\title{
Terahertz out-of-plane coupler based on compact spot-size converter
}

\author{
Hongxiang Zhang (张宏祥) ${ }^{1}$, Changpei Liang (梁昌沛) ${ }^{1}$, Jian Song (宋 健 $)^{1}$, Chenzhong Fu (付陈忠) ${ }^{1}$, \\ Xiaofei Zang (蔵小飞) ${ }^{1}$, Lin Chen (陈 麟) ${ }^{1}$, and Jingya Xie (谢静雅) ${ }^{1,2 *}$ \\ ${ }^{1}$ Terahertz Technology Innovation Research Institute, Terahertz Spectrum and Imaging Technology Cooperative Innovation Center, Shanghai Key Laboratory \\ of Modern Optical System, University of Shanghai for Science and Technology, Shanghai 200093, China \\ ${ }^{2}$ Shanghai Institute of Intelligent Science and Technology, Tongji University, Shanghai 200092, China
}

*Corresponding author: xiejy@usst.edu.cn

Received July 19, 2021 | Accepted August 17, 2021 | Posted Online October 8, 2021

Low-loss dielectric terahertz (THz) chips are efficient platforms for diverse THz applications. One of the key elements in the chip is the coupler. Most of the available THz couplers are in-plane and couple the THz wave from the metal waveguide to the dielectric waveguide. However, out-of-plane couplers are more suitable for wafer-scale testing and tolerant of alignment variation. In this work, we propose an out-of-plane THz coupler for coupling the antenna to the dielectric waveguide. The device is constructed using a grating and a compact spot-size converter. As the conventional optical spot-size converters that apply directly to THz chips are too large, we have designed a compact spot-size converter based on a tapered waveguide with a lens. The total device is $2.9 \mathrm{~cm}$ long and can couple a $7 \mathrm{~mm}$ diameter THz beam to a $500 \mu \mathrm{m}$ wide waveguide. The device can scan the $\mathrm{THz}$ beam, radiate the input rectangular waveguide mode to free space, and drive the rotation angle of the fan beam through the scanning frequency. We fabricated the device using a single lithography step on a silicon wafer. The out-of-plane coupling efficiency was found to be $\sim 5 \mathrm{~dB}$ at $194 \mathrm{GHz}$. The fan-beam steering range was found to be around $40^{\circ}$ in the frequency range of $170-220 \mathrm{GHz}$. The proposed out-of-plane coupling technique may provide an effective way for THz wafer-scale testing with a higher degree of freedom for on-chip integration. Also, the proposed technique being nonmechanical, beam steering using it, may therefore find applications in $\mathrm{THz}$ radar, communication, and sensing.

Keywords: terahertz device; grating coupler; spot-size converter; beam steering.

DOI: 10.3788/COL202220.021301

\section{Introduction}

Integrated terahertz (THz) devices are capable of improving system performance in security imaging, high-speed communication, molecular spectroscopy, and other applications ${ }^{[1,2]}$. Various THz circuits including conventional microstrip lines ${ }^{[3]}$, coplanar waveguides ${ }^{[4,5]}$, substrate-integrated image guides $(\mathrm{SIIGs})^{[6,7]}$, and spoof surface plasmon polariton (SSPP) waveguides $^{[8-13]}$ have been reported. Metallic waveguides suffer from high ohmic losses at the $\mathrm{THz}$ band ${ }^{[14-18]}$. They are costly and difficult to fabricate. Thus, the all-dielectric chips are preferred. As the all-dielectric chips are based on guided-wave optics, they are reasonably efficient. However, it is not possible to independently operate the $\mathrm{THz}$ dielectric functional circuits due to the difficulties in the on-chip generation and detection of $\mathrm{THz}$ radiation. Usually, the dielectric functional circuits are connected to the conventional $\mathrm{THz}$ rectangular metallic waveguides or antennas. Hence, it is necessary to develop the $\mathrm{THz}$ couplers with high efficiency. Recently, different types of $\mathrm{THz}$ couplers have been reported, including inverse-taper waveguides $^{[19]}$, Luneburg lenses ${ }^{[20]}$, and directly coupled couplers ${ }^{[21]}$. All of these couplers work on the principle of coupling the $\mathrm{THz}$ wave through the chip facet in-plane. However, out-of-plane couplers can be used directly to inject and extract $\mathrm{THz}$ waves at arbitrary locations on the chip, which is very important for on-wafer testing. A promising approach is to couple the light to (or from) a chip via a diffraction grating with a spot-size converter formed on the chip surface. This technique is widely used in the optical domain ${ }^{[22-26]}$. Interestingly, the diffraction grating structures have good beam steering properties ${ }^{[27,28]}$. However, this coupler will be very large in size in the $\mathrm{THz}$ frequency range, probably more than $10 \mathrm{~cm}$ in length, due to the large wavelength of the $\mathrm{THz}$ wave. Thus, it will be difficult to integrate the coupler with other $\mathrm{THz}$ functional devices on a single wafer.

In this paper, an out-of-plane coupler for coupling the $\mathrm{THz}$ antenna and planar dielectric waveguide is proposed. The coupler consists of a uniform grating and a compact spot-size 
converter. The $\mathrm{THz}$ antenna used is a standard horn antenna. The antenna length is $26.314 \mathrm{~mm}$, and the aperture size is $9.55 \mathrm{~mm} \times 12.548 \mathrm{~mm}$. To match the incident-beam size, the coupling grating is fabricated on a high-resistivity silicon wafer having the dimension of $1 \mathrm{~cm}$ wide and $1 \mathrm{~cm}$ long. A THz singlemode silicon waveguide is $\sim 500 \mu \mathrm{m}$ wide ${ }^{[21]}$. Hence, a spot-size conversion for $1 \mathrm{~cm}$ and $500 \mu \mathrm{m}$ wide waveguides is required. However, due to the large wavelength of the $\mathrm{THz}$ wave, the traditional taper waveguide is too long to be integrated on a wafer. Spot-size converters with lenses that are compact and efficient to focus the guide mode in the dielectric waveguide have been analyzed theoretically ${ }^{[29]}$. In this work, we have experimentally verified the concept by employing it in our coupler design at the $\mathrm{THz}$ band. The converter is smaller than one-tenth of the traditional taper. The total length of the coupler is $2.9 \mathrm{~cm}$, which results in simpler fabrication on a single silicon wafer. The coupling loss has been found to be $\sim 5 \mathrm{~dB}$ at $194 \mathrm{GHz}$. We also demonstrate the fan-beam steering application of the devices. The steering range of the beam radiated from the grating in the frequency range of $170 \mathrm{GHz}$ to $220 \mathrm{GHz}$ is about $40^{\circ}$. The device is fabricated using a single lithography step on a silicon wafer, which is simple and low cost.

\section{Principle and Design}

Figure 1(a) shows the schematic of the device fabricated on a silicon wafer. The wafer is $470 \mu \mathrm{m}$ thick and etched with a depth of $340 \mu \mathrm{m}$. The device consists of two couplers connected by a ridge waveguide. A $500 \mu \mathrm{m}$ wide ridge waveguide is designed to achieve single-mode operation, which is usually used in integrated devices. Each coupler is formed by a grating and a compact spot-size converter with the lens. One grating couples the $\mathrm{THz}$ wave radiated from a horn antenna into a multi-mode waveguide. The spot-size converter is used to couple the input wave from the multi-mode waveguide to the single-mode waveguide. Another coupler couples the $\mathrm{THz}$ wave back to another horn antenna. As we introduce a lens into the spot-size converter, the length of the taper reduces significantly. Figure 1(b) shows the illustration of the spot-size converter with the lens. The filling factor of each unit can be adjusted to form a waveguide lens and focus the input $\mathrm{THz}$ wave. This coupler can be used as the fan-beam steering device, as shown in Fig. 1(c). The $\mathrm{THz}$ wave is driven from the ridge-shaped waveguide and radiated into free space through the grating structure, and the resulting beam is controlled by frequency scanning.

We first designed the uniform grating, and the principle of the Bragg grating can be expressed as ${ }^{[30]}$

$$
\Lambda=\frac{m \lambda_{0}}{n_{\mathrm{eff}}-\sin \theta}, \quad m=0, \pm 1, \pm 2 \ldots
$$

where $\Lambda$ is the period of the grating, $m$ is the diffractive order, and $n_{\text {eff }}$ and $\theta$ are the effective refractive index and the angle of incidence, respectively. Following Eq. (1), we can calculate theoretical grating parameters for the center frequency of $187 \mathrm{GHz}$. Then, we optimized the grating structure based on finite-difference time-domain (FDTD) solutions from Lumerical using a Gaussian source with a waist radius of $3.5 \mathrm{~mm}$ and an incident angle of $9^{\circ}$. The optimal parameters in the inset of Fig. 2(a) are as follows: grating period $(\Lambda)$ is $0.96 \mathrm{~mm}$, duty cycle $(d / \Lambda)$ is 0.5 , and grating width $L_{1}$ is $1 \mathrm{~cm}$. The number of grating periods is 10. The total grating area is about $1 \mathrm{~cm} \times 1 \mathrm{~cm}$. As shown in Fig. 2(a), the coupling efficiency at $187 \mathrm{GHz}$ is $-4.65 \mathrm{~dB}$, and the $3 \mathrm{~dB}$ bandwidth is $8.5 \mathrm{GHz}$. This coupling efficiency is obtained by calculating $10 \log \left(P_{\text {out }} / P_{\text {in }}\right)$ in the simulation, where $P_{\text {in }}$ is the output power of the Gaussian source, and $P_{\text {out }}$ is the useful power coupling into the waveguide through
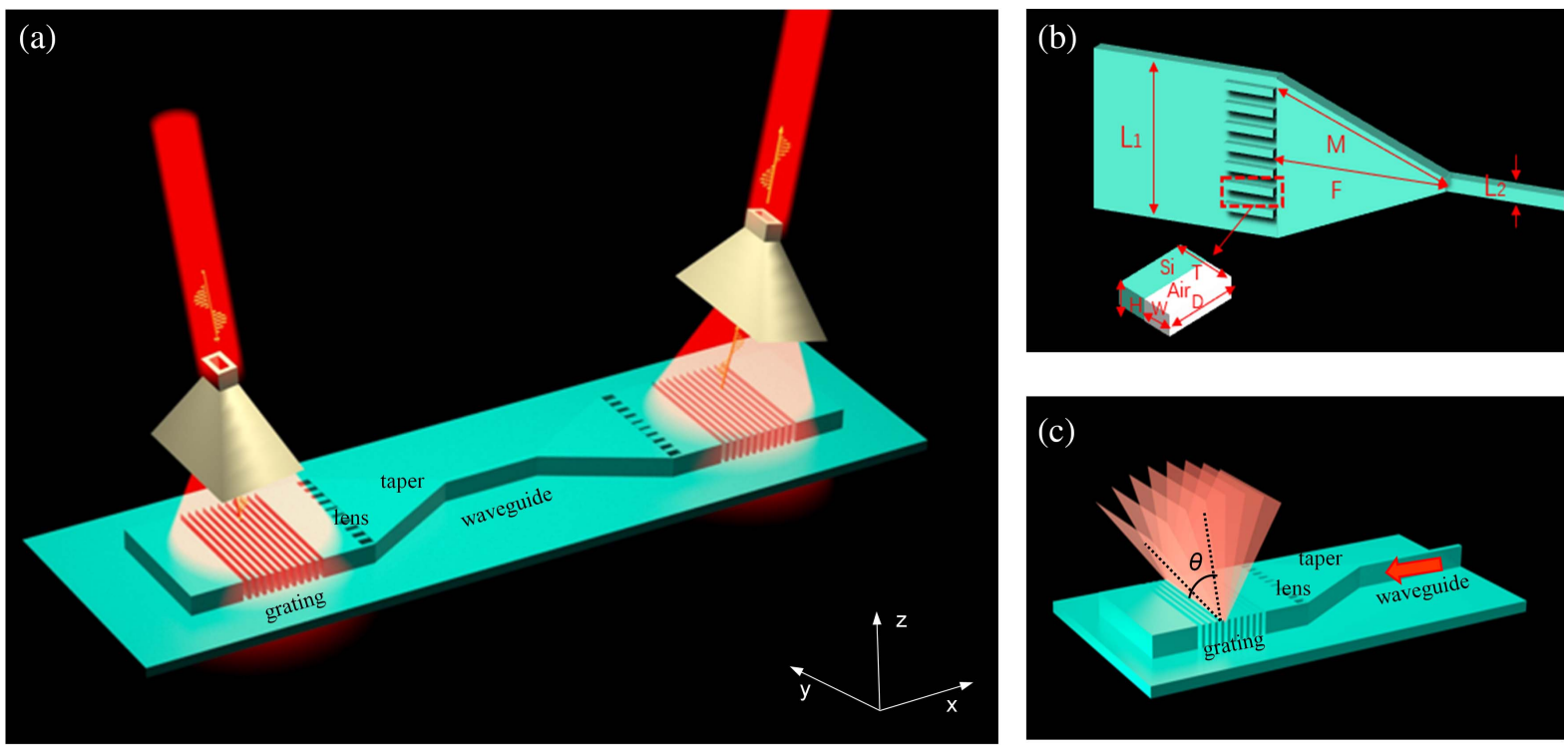

Fig. 1. (a) Schematic of the device consists of two horn antennas, uniform gratings, and spot-size converters with a lens. (b) Illustration of the spot-size converter with a lens. (c) Schematic of the fan-beam steering application. 


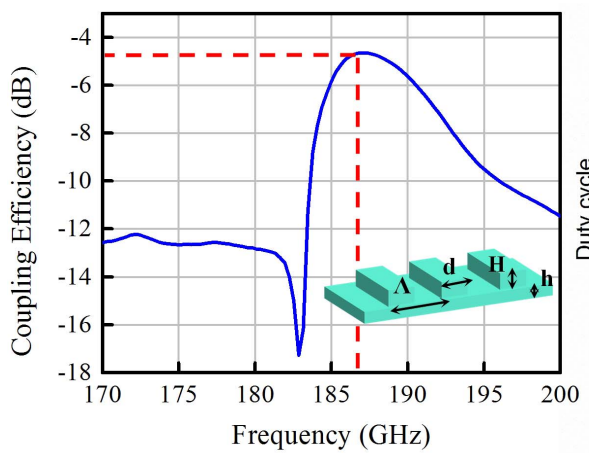

(a)

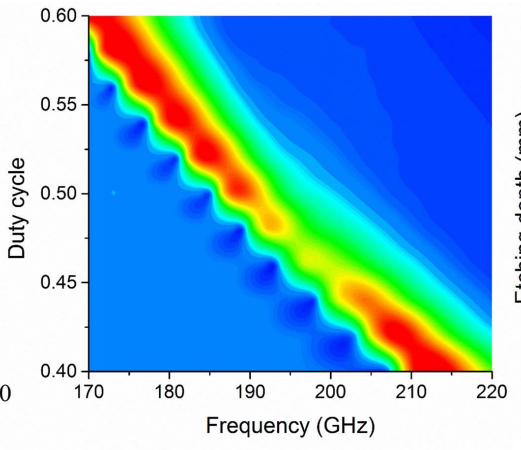

(b)

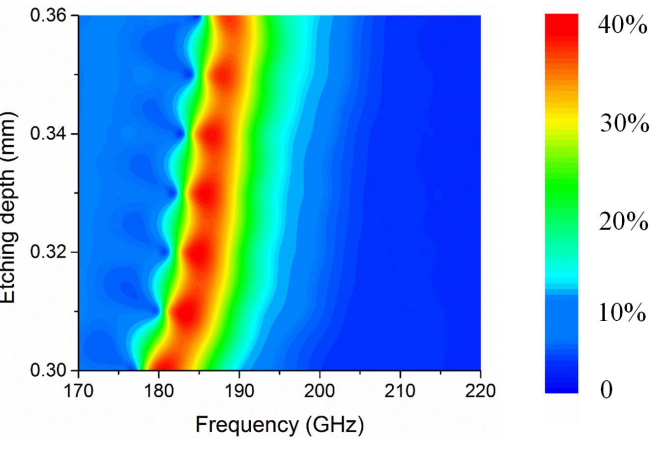

(c)

Fig. 2. (a) Simulated coupling efficiency spectrum of grating. The coupling efficiency for different (b) duty cycles and (c) etching depths.

the grating. According to Eq. (1), the central frequency for different grating parameters can be estimated. However, in order to observe the results more accurately, we also simulate the coupling efficiency for different duty cycles and etching depths. It may be observed from Fig. 2(b) that the central frequency shifts toward a lower frequency, as the duty cycle was increased from 0.4 to 0.6 . We can see some sawtooth in it, and this is because of the large scanning step. The scanning step of the duty cycle is 0.02. Figure 2(c) shows that the central frequency shifts to the higher frequency as the etching depth increases. Because the etching depth is not changed in a large range, the maximum coupling efficiency is not greatly affected.

To reduce the length of the device, we designed a compact and efficient spot-size converter with the lens. The purpose of using (a)

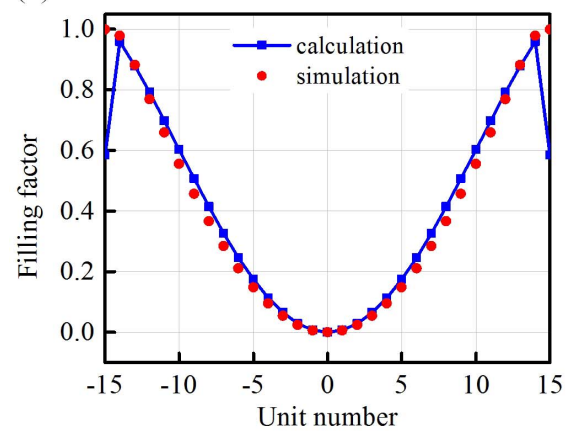

(d) (b)

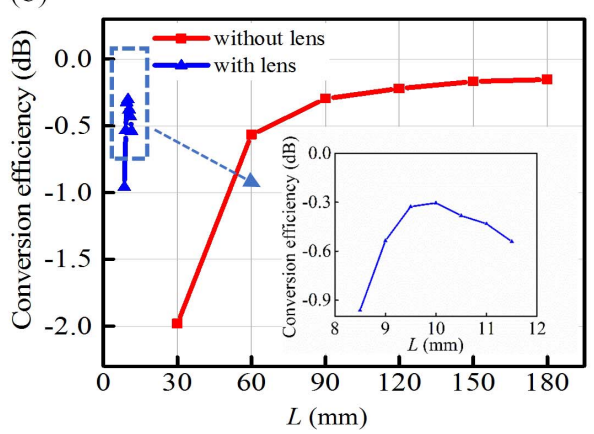

(c)

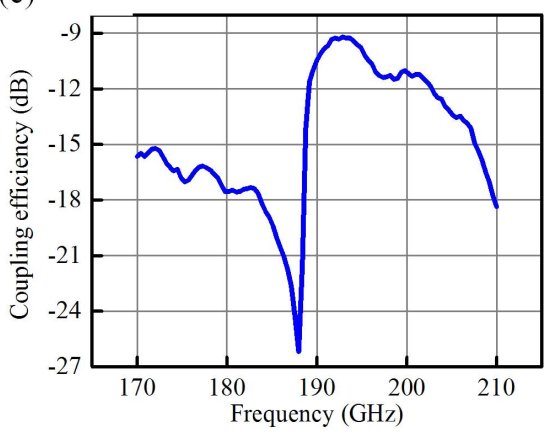

(e)

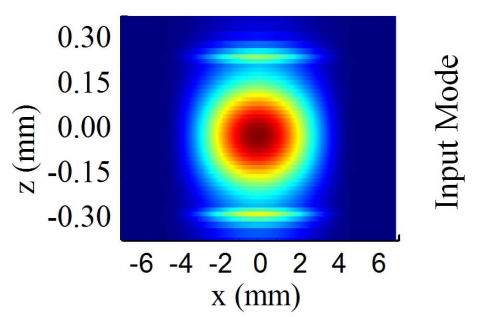

(f)

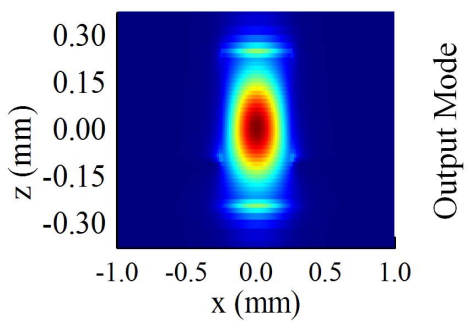

Fig. 3. (a) Calculation and simulation filling factors of each unit. (b) Conversion efficiency of the designed spot-size converter with a lens and the traditional adiabatic converter without lenses of different lengths. (c) Coupling efficiency of the whole structure including two designed couplers connected by a $6 \mathrm{~cm}$ long waveguide. (d) Normalized optical power distribution in the device at $192.857 \mathrm{GHz}$. (e) Input optical field distribution of the spot-size converter. (f) Output optical field distribution of the spot-size converter. 

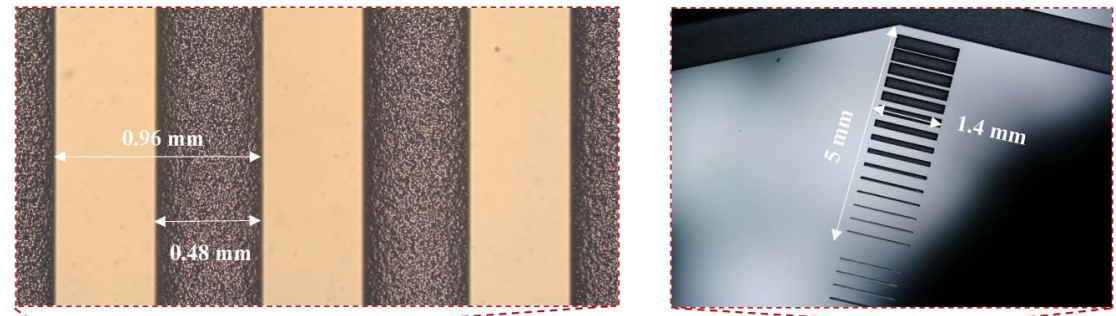

(a)
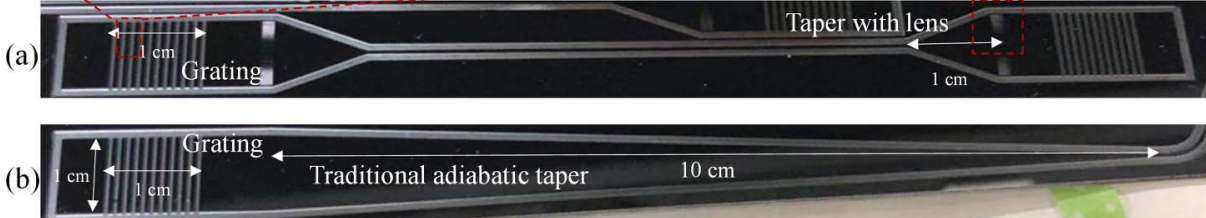

$10 \mathrm{~cm}$

(c)

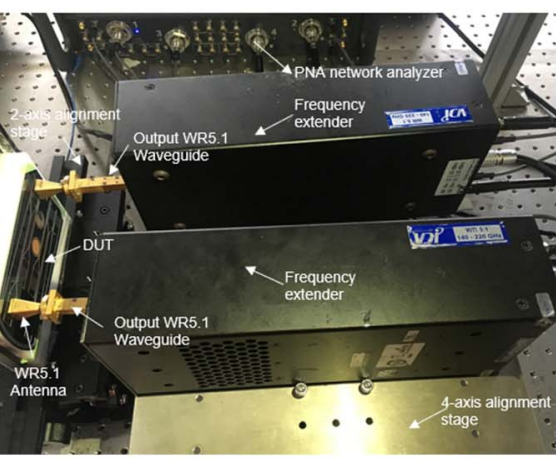

(d)

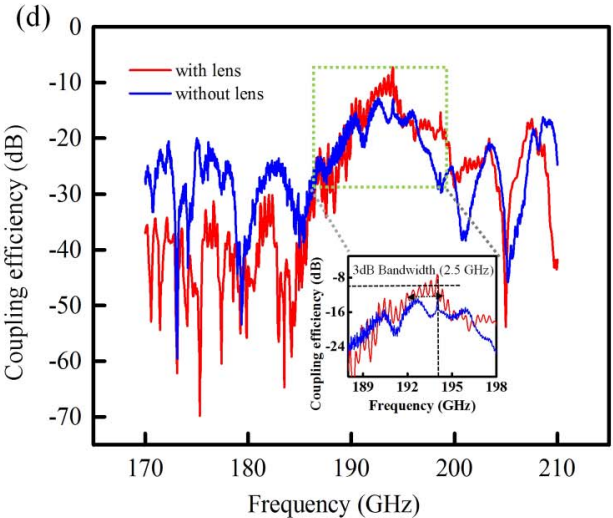

Fig. 4. (a) Optical image of our device with two out-of-plane couplers and a straight waveguide. (b) The optical image of a traditional coupler without a lens. (c) Experimental setup for coupling efficiency measurement of device under test (DUT). (d) Measured normalized coupling efficiency of devices with and without a lens.

the lens is to change the effective refractive index of the structure for phase compensation so that the input $\mathrm{THz}$ wave could be focused on the spot-size converter. As shown in Fig. 1(b), the lens consists of a periodic silicon block and air slits. Each unit has uniform period $(T)$ and lens thickness $(D)$ but different air slit width $(W)$. According to the Fermat principle and effective index method (EIM), the effective index of each unit is approximately determined as follows:

$$
n_{\text {thick }} D+n_{\text {thick }} F=n_{m} D+n_{\text {thick }} M
$$

where $n_{\text {thick }}$ is the effective refractive index of the $470 \mu \mathrm{m}$ planar waveguide, $n_{m}$ is the effective refractive index of the $m$ th unit, which can be seen in Fig. 1(b), $F$ is the distance from the central unit to the focus, and $M$ is the distance from the $m$ th unit to the focus. For all of the units, the light path from the incident light to the focus is equal. The effective refractive index of units can be tuned by the air slit width and approximately calculated by

$$
n_{m}=\sqrt{f_{m} n_{\text {thin }}^{2}+\left(1-f_{m}\right) n_{\text {thick }}^{2}}
$$

where $f_{m}$ is the filling factor of the $m$ th unit, which is determined by the ratio of slit width $(W)$ to period $(T)$. The effective refractive index of the $130 \mu \mathrm{m}$ planar waveguide is $n_{\text {thin }}$.
Following Eqs. (2) and (3), we calculated the approximate filling factors of 31 units, as shown by the blue curve in Fig. 3(a). The period $(T)$ and thickness $(D)$ of the lens were $0.3 \mathrm{~mm}$ and $1.4 \mathrm{~mm}$, respectively. The focal length $(F)$ was $10 \mathrm{~mm}$. We performed a numerical simulation to optimize the filling factors of units and the length of the tapered waveguide at $187 \mathrm{GHz}$. Since the tapered waveguide was employed in the converter, the variation gradient of the filling factor is slightly different from the calculation result, which is shown by the red dots in Fig. 3(a). The conversion efficiency reaches a maximum of $-0.3 \mathrm{~dB}$ when the taper is $10 \mathrm{~mm}$ long, as shown by the blue curve in Fig. 3(b). This conversion efficiency is obtained by calculating $10 \log \left(P_{\text {lens_out }} / P_{\text {lens_in }}\right)$, where $P_{\text {lens_in }}$ is the input power of the lens, and $P_{\text {lens_out }}$ is the output power of the lens. To compare with our device, we also simulated a traditional adiabatic tapered waveguide, as shown by the red curve in Fig. 3(b). The conversion efficiency increases with the taper length. To achieve the same conversion efficiency as our device, the taper length needs to be around $10 \mathrm{~cm}$, which is too long to be integrated on a wafer.

Then, we simulated the whole structure, including two designed couplers connected by a $6 \mathrm{~cm}$ long waveguide. Figure 3(c) shows that the coupling efficiency of the whole structure can reach $-9.2 \mathrm{~dB}$ at $192.857 \mathrm{GHz}$. The $3 \mathrm{~dB}$ coupling bandwidth is about $14 \mathrm{GHz}$. The normalized optical power 
distribution of the device with the lens is shown in Fig. 3(d). Figures 3(e) and 3(f) show the input and output optical field distributions of the spot-size converter, respectively. The optical mode is found to be well concentrated into the signal mode waveguide, and the size of the optical mode is significantly reduced to $\sim 0.5 \mathrm{~mm}$. The simulation results show that the structure size can be reduced after adding the slit silicon lens.

\section{Experimental Results}

The device was fabricated on a 6 inch $(1$ inch $=2.54 \mathrm{~cm})$ highresistivity wafer, which is $470 \mu \mathrm{m}$ thick and double-sided polished. The dielectric constant is 11.7 , and the resistivity is more than $5 \mathrm{k} \Omega \cdot \mathrm{cm}$. The wafer is etched with a depth of $340 \mu \mathrm{m}$ to form ridge waveguides by the deep reactive ion etching (DRIE) process. The optical image of the device with the lens is shown in Fig. 4(a). There are two compact couplers, and the length of the connecting waveguide between them is $6 \mathrm{~cm}$. Figure 4(b) shows the optical image of the traditional coupler without a lens. The coupler structure is $11.9 \mathrm{~cm}$ long, which is obviously hard to integrate with other $\mathrm{THz}$ devices on a 6 inch wafer. Figure 4(c) shows the experimental setup for coupling measurement.

We used an Agilent N5227A PNA network analyzer, a set of WR-5.1 140-220 GHz expanders, and a pair of WR-5.1 horn antennas to characterize the coupling performance of the device. After the calibration, the horn antennas were connected to hollow metallic waveguide ports. Then, we used the four-axis alignment stage to adjust the coupling angle and the distance between horn antennas and gratings. The connected waveguide is $6 \mathrm{~cm}$ long, and the transmission loss of the waveguide in the TM mode was about $0.054 \mathrm{~dB} / \mathrm{cm}^{[21]}$. Therefore, the loss of the connected waveguide is negligible. Notably, the loss of two antennas should be taken into consideration because it could reach about

Table 1. Comparison of Various Terahertz Silicon Waveguide Couplers.

\begin{tabular}{lccccc}
\hline Structure Description & Device Size $\left(\mathrm{mm}^{2}\right)$ & Coupling Efficiency (dB) & Bandwidth $(\mathrm{GHz})$ & Ref. & Note \\
\hline Inverse-taper waveguides & 0.403 & -0.42 & $>60$ & {$[19]$} & In-plane \\
Luneburg lenses & $\sim 368$ & -7 & 70 & {$[20]$} & In-plane \\
Directly coupled coupler & None & -4 & $>20$ & {$[21]$} & In-plane \\
Grating coupler & 290 & -5 & 2.5 & This work & Out-of-plane \\
\hline
\end{tabular}
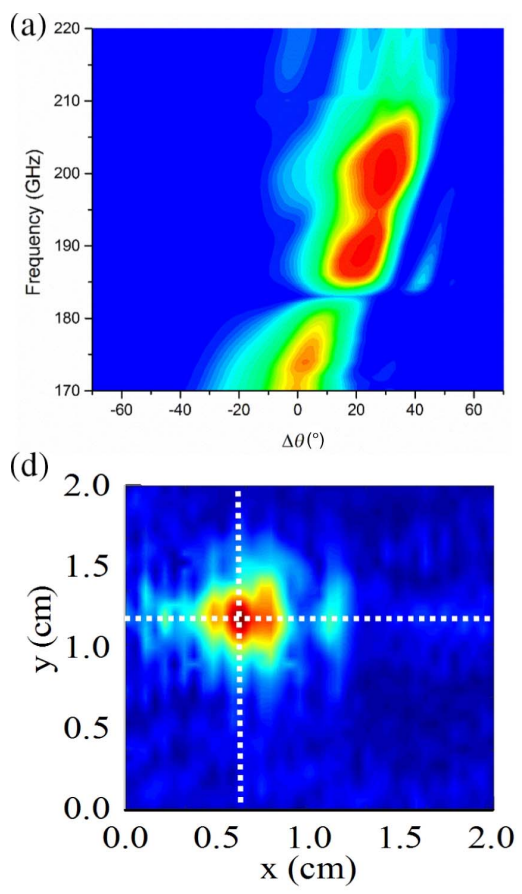

(b)

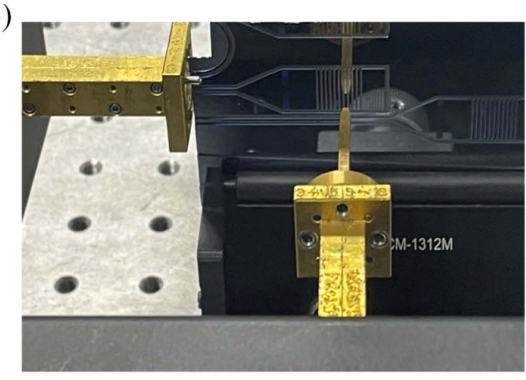

(e)

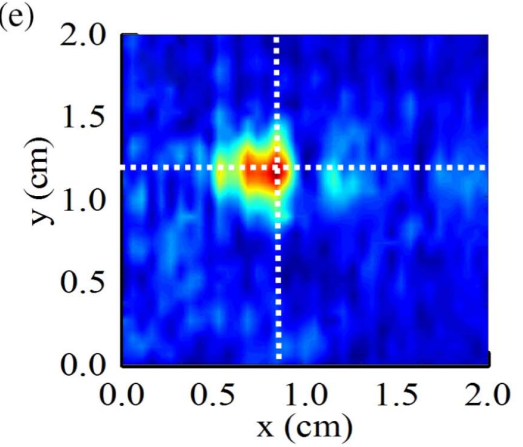

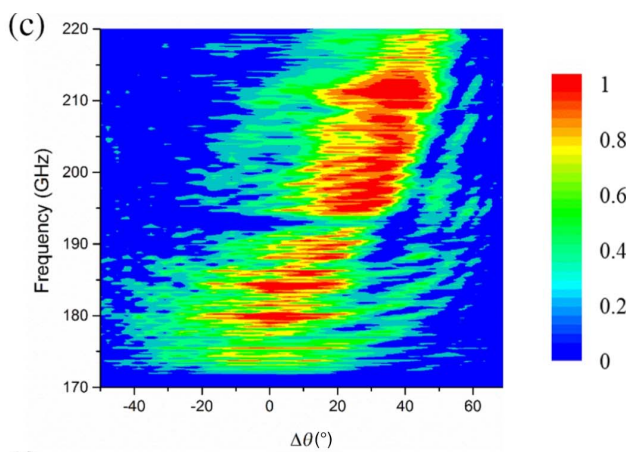

(f) 2

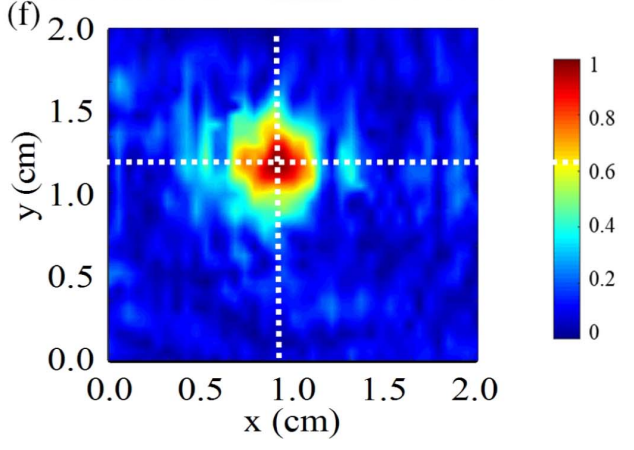

Fig. 5. (a) Simulated electric field steering distribution of the beam for frequency variation. (b) The experimental setup of the fan-beam steering. (c) The measured electric field steering distribution of the beam for frequency variation. Measured electric field distributions of the beam at different frequencies of (d) $180 \mathrm{GHz}$, (e) $190 \mathrm{GHz}$, and (f) $200 \mathrm{GHz}$. 
$9 \mathrm{~dB}$. Under this situation, we normalized the test results, and the measured normalized coupling efficiency of the device using couplers with the lens is shown in Fig. 4(d) by the red curve. It is observed that the maximal transmission reaches $-10 \mathrm{~dB}$. The coupling efficiency of one coupler proposed in this paper is around $-5 \mathrm{~dB}$. Compared with the simulation results, the center frequency is shifted to $194 \mathrm{GHz}$, and the $3 \mathrm{~dB}$ bandwidth is reduced to $2.5 \mathrm{GHz}$. We also measured the coupling efficiency of the coupler without a lens, as shown in Fig. 4(d) by the blue curve. Due to the long spot-size converter and connected waveguide, the maximal coupling efficiency of this coupler could only reach $-6.5 \mathrm{~dB}$. The results indicate that our device is extremely compact and has a lower loss. The coupling efficiency of our coupler is close to that of the uniform grating devices in optical bands ${ }^{[31]}$, and special designs can be used to improve the coupling efficiency in the future ${ }^{[32]}$. Table 1 lists the comparison of our device with various reported $\mathrm{THz}$ couplers including inverse-taper waveguides, Luneburg lenses, and directly coupled couplers. The main advantage of our work is that it provides an out-of-plane coupling in the $\mathrm{THz}$ band.

Finally, we demonstrated the fan-beam steering application of the grating. The illustration of the beam steering application has been shown in Fig. 1(c). According to Eq. (1), the angle of the diffraction light beam $(\theta)$ will increase with the frequency. Numerical simulation results show that within the frequency range of $170-220 \mathrm{GHz}$, the steering range of the near-field beam can reach $\sim 40^{\circ}$ in the $\theta$ direction, as shown in Fig. 5(a). Figure 5 (b) shows the experimental setup. The $\mathrm{THz}$ wave is coupled endto-end into the ridge-shaped silicon waveguide, and a rectangular waveguide probe is used as a receiver to obtain the electric field distribution of the beam radiated from the grating. The distance between the probe and the grating is $5 \mathrm{~mm}$, and the $x-y$ plane scan range is $2 \mathrm{~cm} \times 2 \mathrm{~cm}$, which resulted in $39 \times 39$ pixels. As shown in Fig. 5(c), the experimental fan-beam steering range is around $40^{\circ}$ in the frequency range of $170-220 \mathrm{GHz}$. We show the normalized beam profiles of the electric field distributions of $180 \mathrm{GHz}, 190 \mathrm{GHz}$, and $200 \mathrm{GHz}$ in Figs. 5(d), 5(e), and 5(f), respectively. It can be seen that the electric field is mainly distributed at $y=1.2 \mathrm{~cm}$, i.e., right in the middle of the grating. The radiated beam moves to the right along the $x$ axis as the frequency increases, i.e., the steering angle increases. Note that the electric field steering distribution in Fig. 5(c) was extracted from the electric field of each beam of a different frequency at $y=1.2 \mathrm{~cm}$. Thus, $\mathrm{THz}$ beam steering application could be obtained using the device.

\section{Conclusion}

In this paper, we proposed and demonstrated a $\mathrm{THz}$ out-ofplane coupler with a compact spot-size converter. The total length of the device is $2.9 \mathrm{~cm}$, which is much shorter than the coupler with a traditional spot-size converter. Experimental results show that the coupling efficiency is $\sim 5 \mathrm{~dB}$ at $194 \mathrm{GHz}$, and the beam steering range is about $40^{\circ}$ from $170 \mathrm{GHz}$ to $220 \mathrm{GHz}$. Therefore, the devices provide out-of-plane coupling and fan-beam steering of $\mathrm{THz}$ dielectric chips. We believe that the proposed structure will contribute to the development of $\mathrm{THz}$ integrated devices, especially silicon-based devices.

\section{Acknowledgement}

This work was supported in part by the National Key Research and Development Program of China (No. 2017YFA0701005), the National Natural Science Foundation of China (Nos. 61705131, 61671302, and 61871268), and the Major National Development Project of Scientific Instrument and Equipment (No. 2016YFF0100503).

\section{References}

1. H. Heinz-Wilhelm, "Towards THz integrated photonics," Nat. Photon. 4, 503 (2010).

2. E. Seok, D. Shim, C. Y. Mao, R. N. Han, S. Sankaran, C. H. Cao, W. Knap, and K. O. Kenneth, "Progress and challenges towards terahertz CMOS integrated circuits," IEEE J. Solid-St. Circ. 45, 1554 (2010).

3. E. Peytavit, C. Donche, S. Lepilliet, G. Ducournau, and J. F. Lampin, "Thinfilm transmission lines using cyclic olefin copolymer for millimetre-wave and terahertz integrated circuits," Electron. Lett. 47, 453 (2011).

4. P. Focardi, A. Neto, and W. R. Mcgrath, "Coplanar-waveguide-based terahertz hot-electron-bolometer mixers: improved embedding circuit description," IEEE Trans. Microwave Theory 50, 2374 (2002).

5. L. Cao, A. S. Grimault-Jacquin, and F. Aniel, "Comparison and optimization of dispersion, and losses of planar waveguides on benzocyclobutene (BCB) at $\mathrm{THz}$ frequencies: coplanar waveguide (CPW), microstrip, stripline and slotline," Prog. Electromagn. Res. 56, 161 (2013).

6. K. Wu, Y. J. Cheng, T. Djerafi, and W. Hong, "Substrate-integrated millimeter-wave and terahertz antenna technology," Proc. IEEE 100, 2891 (2012).

7. A. Patrovsky and $\mathrm{K}$. Wu, "94-GHz planar dielectric rod antenna with substrate integrated image guide (SIIG) feeding," IEEE Antenn. Wirel. Pr. 5, 435 (2006).

8. K. Song and P. Mazumder, "Active terahertz spoof surface plasmon polariton switch comprising the perfect conductor metamaterial," IEEE Trans. Electron. Dev. 56, 2792 (2009).

9. Z. Xu, K. Song, and P. Mazumder, "Analysis of doubly corrugated spoof surface plasmon polariton (DC-SSPP) structure with sub-wavelength transmission at THz frequencies," IEEE Trans. Terahertz Sci. Technol. 2, 345 (2012).

10. H. Z. Yao, S. C. Zhong, and W. L. Tu, "Performance analysis of higher mode spoof surface plasmon polariton for terahertz sensing," J. Appl. Phys. 117, 133104 (2015).

11. Y. J. Guo, K. D. Xu, and X. H. Tang, "Spoof plasmonic waveguide developed from coplanar stripline for strongly confined terahertz propagation and its application in microwave filters," Opt. Express 26, 10589 (2018).

12. K. Song and P. Mazumder, "Dynamic terahertz spoof surface plasmonpolariton switch based on resonance and absorption," IEEE Trans. Electron. Dev. 58, 2172 (2011).

13. C. R. Williams, S. R. Andrews, S. A. Maier, A. I. Fernández-Domínguez, L. Martín-Moreno, and F. J. García-Vidal, "Highly confined guiding of terahertz surface plasmon polaritons on structured metal surfaces," Nat. Photon. 2, 175 (2008).

14. M. Nagel, A. Marchewka, and H. Kurz, "Low-index discontinuity terahertz waveguides,” Opt. Express 14, 9944 (2006).

15. P. A. George, C. Manolatou, F. Rana, A. L. Bingham, and D. R. Grischkowsky, "Integrated waveguide-coupled terahertz microcavity resonators," Appl. Phys. Lett. 91, 191122 (2007).

16. J. Tao, B. Hu, X. Y. He, and Q. J. Wang, "Tunable subwavelength terahertz plasmonic stub waveguide filters," IEEE Trans. Nanotechnol. 12, 1191 (2013).

17. S. Atakaramians, S. Afshar, T. M. Monro, and D. Abbott, "Terahertz dielectric waveguides," Adv. Opt. Photon. 5, 169 (2013). 
18. Y. Yang, Y. Yamagami, X. Yu, P. Pitchappa, and R. Singh, "Terahertz topological photonics for on-chip communication," Nat. Photon. 14, 446 (2020).

19. N. Ranjkesh, M. Basha, A. Taeb, and N. S. Safavi, "Silicon-on-glass dielectric waveguide-Part II: for THz applications," IEEE Trans. Terahertz Sci. Technol. 5, 280 (2015).

20. D. Headland, W. Withayachumnankul, R. Yamada, M. Fujita, and T. Nagatsuma, "Terahertz multi-beam antenna using photonic crystal waveguide and Luneburg lens," APL Photon. 3, 126105 (2018).

21. J. Y. Xie, X. Zhu, X. F. Zang, Q. Q. Cheng, L. Chen, and Y. M. Zhu, “Terahertz integrated device: high- $Q$ silicon dielectric resonators," Opt. Mater. Express 8, 50 (2018).

22. F. V. Laere, T. Claes, J. Schrauwen, S. Scheerlinck, W. Bogaerts, D. Taillaert, L. O'Faolain, D. V. Thourhout, and R. Baets, "Compact focusing grating couplers for silicon-on-insulator integrated circuits," IEEE Photon. Tech. Lett. 19, 1919 (2007).

23. R. Halir, A. Ortega-Monux, J. H. Schmid, C. Alonso-Ramos, J. Lapointe, D. X. Xu, J. G. Wangüemert-Pérez, I. Molina-Fernandez, and S. Janz, "Recent advances in silicon waveguide devices using sub-wavelength gratings,” IEEE J. Sel. Top. Quantum. 20, 8201313 (2013).

24. W. D. Sacher, Y. Huang, L. Ding, J. F. Taylor, H. Jayatilleka, G. Q. Lo, and J. K. S. Poon, "Wide bandwidth and high coupling efficiency $\mathrm{Si}_{3} \mathrm{~N}_{4}$-on-SOI dual-level grating coupler," Opt. Express 22, 10938 (2014).
25. R. Y. Ge, H. Li, Y. Han, L. Chen, J. Xu, M. Y. Wu, Y. Q. Li, Y. N. Luo, and $\mathrm{X}$. L. Cai, "Polarization diversity two-dimensional grating coupler on $x$-cut lithium niobate on insulator," Chin. Opt. Lett. 19, 060006 (2021).

26. H. Ma, H. T. Yang, B. Tang, M. L. Wei, J. Y. Li, J. H. Wu, P. Zhang, C. L. Sun, L. Li, and H. T. Lin, "Passive devices at $2 \mu \mathrm{m}$ wavelength on $200 \mathrm{~mm}$ CMOScompatible silicon photonics platform [Invited]," Chin. Opt. Lett. 19, 071301 (2021)

27. K. Keisuke, T. Tomoki, H. Shoji, A. Hiroshi, K. Fumio, and B. Toshihiko, "Fan-beam steering device using a photonic crystal slow-light waveguide with surface diffraction grating," Opt. Lett. 42, 4990 (2017).

28. J. K. Doylend, M. J. R. Heck, J. T. Bovington, J. D. Peters, L. A. Coldren, and J. E. Bowers, "Two-dimensional free-space beam steering with an optical phased array on silicon-on-insulator," Opt. Express 19, 21595 (2011).

29. J. J. Zhang, J. B. Yang, H. Xin, J. Huang, D. B. Chen, and Z. J. Zhang, "Ultrashort and efficient adiabatic waveguide taper based on thin flat focusing lenses," Opt. Express 25, 19894 (2017).

30. S. Nambiar and S. K. Selvaraja, "High-efficiency broad-bandwidth subwavelength grating based fibre-chip coupler in SOI," Opt. Eng. 57, 017115 (2018).

31. D. Taillaert, F. V. Laere, M. Ayre, W. Bogaerts, D. V. Thourhout, P. Bienstman, and R. Baets, "Grating couplers for coupling between optical fibers and nanophotonic waveguides,” Jpn. J. Appl. Phys. 45, 6071 (2006).

32. D. Taillaert, P. Bienstman, and R. Baets, "Compact efficient broadband grating coupler for silicon-on-insulator waveguides," Opt. Lett. 29, 2749 (2004). 